\title{
HIP ADDUCTOR MUSCLE RELEASE IN THE TREATMENT OF CHILDREN WITH CERERRAL PALSY
}

\author{
BEVERLEY TRAUB, B.Sc. (Physiotherapy) (Witwatersrand)* \& COLLEEN PITCHFORD, B.Sc. (Physiotherapy) \\ (Witwatersrand)*
}

The aims of this study are:

- to describe the postoperative management of adductor muscle release patients.

- to formulate a detailed postoperative physiotherapy programme to enable accurate evaluation and prognostication.

In a retrospective study we have shown that the results achieved using this procedure and postoperative management programme are very satisfactory in terms of the success rate and end results.

This operation is performed on cerebral palsied children who are incapacitated due to marked spasticity of the hip adductor muscles, which results in a scissoring gait on attempted ambulation, as well as reduced abduction range which may be so severe as to cause subluxation of the hips. This subluxation normally occurring in children with less than $30^{\circ}$ of totalabduction may progress to frank dislocation and its attendant complications. Thus, the indications for this procedure may be formulated as follows:

- scissor gait with ambulatory impairment

- threatened subluxation or imminent dislocation

- difficulty in nursing the severely spastic child.

\section{SURGICAL PROCEDURE}

The success of the operation is dependent on a number of factors:

Age of the patient

Normatly the operation is performed on children between the ages of four and six years for practical purposes, i.e. difficulty in the adequate assessment of abduction strength in a child of under three years, understanding and interpretation by the child etc.

*Done whilst physiotherapists at Natalspruit Hospital. Received 26 October 1981.
Home and family background

This is known to have an effect on postoperative management. but in our short study all the children were of similar background (boarding school) and this parameter was unassessed.

\section{Adequale postoperative care}

Adequate postoperative care implies satisfactory analgesia, motivated nursing staff and antibiotic cover.

Postoperatively the child is immobilised in a plaster spica for 3-4 weeks. The cast is applied with the hips in full abduction and extension with $10^{\circ}-15^{\circ}$ external rotation. Toe to groin casts joined by an abduction bar should not be used as they invariably lead to pelvic obliquity. Approximately, two days postoperatively the Portovac catheters ard removed, provided they are no longer draining. Should there be no complications, the patient is discharged approximately two weeks later in the care of their parents with instructions regarding follow-up eare. In our study all the children were resident at the Ezebeleni Home for disabled children and thus remained in hospital during the three weeks of immobilisation.

Following removal of the cast the wound is inspected. Should the wound be septic or partly open, treatment in the hydrotherapy pool (a major part of the physiotherapy treatment) is obviously contra-indicated. An alternate programme for these septic cases must be adopted.

\section{PHYSIOTHERAPY}

\section{Week 1}

Pain in the hips and knees was found to be a constant problem in the treatment of these cases.

Hydrotherapy was used for the first 3-5 days and this resulted in good pain relief and allowed freer movement. The pool programme followed was:

- Passive movements of the hips, knees and ankles with special attention to maintaining hip abduction as well as 
gaining full range of knee and ankle joints. (Initially it may be difficult to achieve a full range of movement and no forceful manipulation should be emploved: usually however, by the third day of treatment, the requisite range of movement had been achieved.)

- Assisted/active exercises. These should include:

Supine:

- Hip abduction and adduction

- Hip flexion - straight leg raising

- drawing knees to the chest

- Hip adduction - squeezing a ball between the legs

Prone:

- Hip extension

- Knee flexion and extension

Silling:

- Ankle dorsiflexion and plantarflexion

- Knee flexion and extension over the edge of the step (in the pool)

Side ling:

- Hip adduction and abduction

Kneeling:

- Perform weight transference from one knee to the other Sianding:

- Water waist high: alternate hip flexion through to extension

- Attempted walking.

\section{Week 2}

Once the functional range of the weight-bearing joints is at tained and active exercises a re being adequately performed by the patient, standing and walking, under supervision, in the paralle! bars are allowed.

One immediate problem presented at this point. The children persistently flexed at the knees when attempted weight bearing through the hip joint produced pain. Despite concerted attempts to prevent such knee flexion (which conceivably could lead to fixed flexion deformity (FFD) of the knees) it was unsuccessful. (This pain may be due to a number of factors, such as incisional pain which may be present for variable periods or pain from the immobilised joints due to osteoporosis and joint stiffness.) Analgesia was not used because of the logistics of administration and timing to achieve maximal effect at the time of physiotherapy, and the ever present danger of dependence.

We attempted to prevent knee flexion using backslabs, but found that adequate knee extension was impossible to maintain. For these reasons, the children were placed into cylinder plasters. This forced the child to bear weight through the hips with the knees extended. There was immediate improvement in the gait. During this time maintenance exercises continued (refer to the exercises performed in the pool; now performed on a mat), as well as daily passive stretching of the adductors.

Once the child was walking well (4 - 10 days), the cylinder plasters were removed. It was found that flexion of the knees did not recur and the gait remained acceptable. Depending on the rate of progress, the children were placed in rollator walkers or on crutches.

\section{Week 3-4}

During this phase it was found that all the children were either in walkers, on crutches or walking unaided. There was no problem in motivating the patients and the transition from walker to crutches to unaided ambulation was easily accomplished.

\section{After 4 weeks}

After the initial intensive four-week programme of daily physiotherapy, the child only attended three times a week for up to six months. During this period, the children were all followed up for evidence of contractures. In one case this occurred and night splints were used to treat possible flexion deformities of the knees due to tight hamstring muscles.

\section{CONCLUSION}

Of the five patients studied, three were able to attain unaided gait and this was achieved within four weeks from the beginning of active physiotherapy. (This was due to their less severely spastic condition as all were spastic diplegics.) The other two patients could not walk unaided due to disabling spasticity in one case and triplegia in the other.

Success is attributed to:

- daily physiotherapy

- daily passive stretching of the adductors

- maintenance of adduction range

- the fact that the children were encouraged to sleep prone. Because of the above it was found unnecessary to employ night splints for maintaining abduction.

\section{Bibliography}

Craig J. J. (1967). Cerebral palsy. Modern trends in Orthopaedics 5, 44-69.

Salter R. E. (1970). Textbook of disorders and injuries of the musculoskeletal system. Williams and Wilkins. pp. 97-106.

Sharrard W. J. W. (1973). Paediatric orthopaedics and fractures. Blackwell, pp. 545-549.

Tachdjian M. O. (1972). Paediatric orthopaedics. Saunders. pp. 811-815.

\section{FIRST EUROPEAN CONFERENCE ON RESEARCH IN REHABILITATION 6-8 APRIL 1983}

The First European Conference on Research in Rehabilitation will be held in Edinburgh from 6-8 April. It is being organised by the Rehabilitation Studies Unit of the University of Edinburgh on behalf of the Society for Research in Rehabilitation (of Great Britain). The theme of the Conference will be Measurement of Outcome in Rehabilitation. Contributions will be welcome from all the professions concerned with research into the care of disabled people; doctors, physiotherapists, occupational therapists, rehabilitation engineers, nurses, clinical psychologists, social workers etc. The Conference will be held at the University of Edinburgh and accommodation has been reserved for delegates and accompanying persons in the University's Halls of Residence.

A full cultural and social programme will be arranged as well as visits to clinical facilities. The Conference language will be English.

Secretariat:

Centre for Industrial Consultancy \& Liaison

University of Edinburgh

16 George Square

Edinburgh EH 8 9LD

Scotland, UK

Tel: 03I-667 1011 ext. 2369

Telex: 727442 (UNIVED G) 